\title{
Regard socio-anthropologique sur la géologie de terrain en sciences de la Terre
}

\author{
Laurence Raineau* \\ Socio-anthropologie, Université Paris 1-Panthéon-Sorbonne, Cetcopra, Paris, France
}

Reçu le 29 novembre 2018. Accepté le 16 janvier 2020

«La science en train de se faire » est l'objet d'études peu nombreuses, que ce texte de Laurence Raineau vient compléter en proposant un regard intéressant et nouveau sur le travail des géologues de terrain. L'approche anthropologique se justifie pleinement pour analyser leurs pratiques, ainsi que le langage qu'ils utilisent pour décrire celles-ci. L'étude révèle que ces géologues ont une approche très phénoménologique du terrain alors que l'on pourrait s'attendre à une certaine distanciation par rapport à leur objet d'étude. Cela soulève plusieurs questions, notamment celles de la position du chercheur et de sa conception de son environnement pour traiter du rapport à la « nature », y compris au sein d'une même discipline, géologues de terrain et de laboratoire pouvant sur ces points avoir des approches différentes.

La Rédaction

\begin{abstract}
Résumé - Si dans le contexte environnemental actuel il devient difficile d'identifier une nature autonome et indépendante de l'Homme et de la société, quel est alors l'objet des sciences de la nature ? Nous proposons d'apporter ici une réponse à partir d'une enquête socio-anthropologique réalisée dans le cadre d'un projet interdisciplinaire à l'initiative des sciences de la Terre. Celle-ci nous a conduit à pointer l'importance du rapport au terrain dans la culture du géologue, même si ce terrain est souvent mis au second plan (ou disparaît même parfois) des publications en sciences de la Terre. Cette approche socio-anthropologique de la géologie, révélant la place du sujet dans la formation de la connaissance, permettra de conclure sur l'importance d'une réflexion pluridisciplinaire sur l'ethos du chercheur face aux enjeux environnementaux d'aujourd'hui.
\end{abstract}

Mots-clés : environnement / ressources naturelles / socio-anthropologie / géologie / interdisciplinarité

\begin{abstract}
A socio-anthropological perspective on field geology in Earth sciences. The environmental problems and the climate issue point to the responsibility of human activities in the disturbances of the global ecosystem. It appears that Earth's changes can no longer be set apart from those of society, as expressed by the notion of Anthopocene. Researchers in the natural and social sciences are now induced to work together on issues that have become shared. In this context I was asked as a sociologist to join an interdisciplinary team of researchers in Earth sciences, chemistry and engineering, to work on a project aiming to produce non-carbon hydrogen from waste of the iron and steel industry. This paper is based on fieldwork conducted as part of this project. Its purpose is to question what it is that natural sciences, in this case geology, are studying if we consider that an autonomous nature, independent of humans and of society, does not exist. Observation of geologists at work, mainly on their research field in the Alps, and the interviews I had with them, has led me to point out the intimate relationship that links them to the environment they are studying. This environment has little to do with some abstract natural world or with the global environment such as that of climate change for example. This definition of what is at stake in Earth sciences will impact on the understanding of environmental problems and on the collaboration between the natural and the social sciences that it implies nowadays.
\end{abstract}

Keywords: environment / natural ressources / socio-anthropology / geology / interdisciplinarity

$\overline{\text { *Auteure correspondante }}:$ laurence.raineau@univ-paris1.fr 
La problématique environnementale, qui pointe aujourd'hui les activités humaines comme responsables des perturbations de l'écosystème terrestre ${ }^{1}$, conduit à ne plus pouvoir dissocier les changements et devenirs de la Terre de ceux de la société. Les différentes échelles de temps qui caractérisaient chaque discipline semblent s'écraser pour s'unifier dans le temps de l'Anthropocène $^{2}$. Naomi Oreskes (2015) montre que les sciences de la Terre (en particulier l'hydrologie, la climatologie, la sismologie et la vulcanologie) sont depuis longtemps conscientes des interactions entre systèmes physiques et systèmes sociaux, et pas seulement dans le sens où les catastrophes naturelles ont des incidences sur les Hommes et les sociétés. Elles intégraient déjà, avant même le récent brouillage des frontières entre sciences naturelles et sciences sociales qu'induit l'Anthropocène, des paramètres relatifs aux institutions et aux techniques humaines dans leurs modèles prédictifs, mais souvent sans pouvoir tenir compte des différences majeures entre systèmes physiques et systèmes sociaux ${ }^{3}$. La prise de conscience de ces interdépendances aujourd'hui dans l'ensemble de la société (et dans les sciences humaines) conduit au développement de nombreux projets interdisciplinaires. Les recherches sur les transformations bio-géo-chimiques de la Terre doivent d'ailleurs souvent associer un volet social à leur projet tellement les phénomènes naturels et sociaux paraissent indémêlables.

C'est dans ce contexte qu'une étude ${ }^{4}$ menée par le laboratoire de sciences de la Terre (ISTerre) de Grenoble a ouvert son champ de recherche à un questionnement social, m'amenant à y participer ${ }^{5}$. Le projet, qui réunissait des laboratoires de sciences de la Terre, de chimie, de biologie, de physique et de mécanique, proposait de s'inspirer de processus géochimiques actifs au niveau de certaines rides médio-océaniques pour mettre au point de nouvelles techniques de production

\footnotetext{
${ }^{1}$ Ce que désigne la notion d'Anthropocène, que ce dernier soit considéré comme une nouvelle ère géologique ou comme une «période» de rupture ou de crise. Voir Bonneuil et Fressoz (2013).

${ }^{2}$ C'est ce que Bernadette Bensaude-Vincent exprime par «le clash des temporalités » (Bensaude-Vincent, 2016).

${ }^{3} \mathrm{~N}$. Oreskes pointe en revanche la prise de conscience tardive des sciences historiques qui n'ont pas perçu ce tournant des sciences de la Terre. Elle insiste sur l'urgence de ne plus penser séparément les systèmes physiques et les systèmes sociaux, et propose de commencer par parler de «systèmes sociophysiques » (Oreskes, 2015, p. 264).

${ }^{4}$ Le projet, nommé Géo-Inspire, s'est déroulé de 2013 à 2016 dans le cadre du programme interdisciplinaire du CNRS - Défi ENRS (Transition énergétique : ressources, société, environnement).

${ }^{5}$ L'analyse proposée ici doit d'ailleurs beaucoup aux échanges avec Fabrice Brunet, directeur d'équipe à ISTerre et coordinateur du projet Géo-Inspire.
}

d'hydrogène. L'objectif était double: proposer une nouvelle source d'énergie peu émettrice de $\mathrm{CO}_{2}$ et valoriser des déchets ou sous-produits de l'activité industrielle, comme ceux issus de la production sidérurgique (en l'occurrence les laitiers ${ }^{6}$ ), à partir desquels serait produit l'hydrogène. Il s'agissait donc non seulement de répondre aux exigences de la transition énergétique, mais aussi de développer une nouvelle méthode de valorisation des déchets ou sous-produits d'origine industrielle, actuellement stockés ou faiblement valorisés. Toutefois, ce n'était pas pour accompagner le processus d'optimisation des techniques mises en œuvre (et étudier les aspects économiques ou sociaux en jeu) que les sciences sociales étaient convoquées, mais dans un but réflexif visant à comprendre en quoi l'approche adoptée, qualifiée de "géo-inspirée », exprimait un changement de posture du géologue face à la nature. Considérée comme source d'inspiration, la nature ne serait, dès lors, plus appréhendée comme un ensemble de ressources à exploiter ou de processus à piloter.

Ce questionnement du géologue sur sa posture ne participait-il pas à l'interrogation plus générale dans la société contemporaine sur le dualisme nature/culture? Telle était à peu près la question qui accompagnait mes premiers entretiens et observations lorsque j'ai suivi les géologues sur leur terrain dans les Alpes. Elle dut très vite être reformulée tant il m'est apparu que la relation qu'ils avaient à leur objet ne traduisait en rien une représentation (même en questionnement) d'une nature distanciée et extérieure, en attente d'interprétation. Il semblait que dans leur pratique scientifique ils n'avaient jamais opéré ce grand partage entre eux et la «nature» qu'ils étudiaient ${ }^{\top}$.

C'est donc de ce rapport du géologue à son objet qu'il sera question ici. Rompant avec l'idée que l'objectivité scientifique tient à la distance que le chercheur maintient avec l'objet immédiat, nous défendrons que c'est au contraire l'implication de toute sa personne qui rend possible son savoir ${ }^{8}$. Claude Lévi-Strauss (1962, p. 54) soutenait déjà que «le savoir théorique n'est pas incompatible avec le sentiment, que la connaissance

\footnotetext{
${ }^{6}$ Les laitiers sont des sous-produits solides (des scories) issus de la fusion du métal en sidérurgie. Ils sont principalement composés de silicates, de chaux et d'aluminates.

${ }^{7}$ Ils n'avaient en ce sens sans doute «jamais été modernes» dans leurs pratiques scientifiques pour reprendre le titre du livre de Latour (1991), la modernité s'étant élevée, selon l'auteur, sur une représentation duale du monde entre nature et société.

${ }^{8}$ Si cette position semble partagée dans les sciences de la nature depuis le premier tiers du $\mathrm{XX}^{\mathrm{e}}$ siècle, comme en atteste l'étude de Daston et Galison (2012) sur la construction des images des atlas scientifiques, elle est toujours sujette à discussion en sciences humaines (Dubey, 2013).
} 
peut être à la fois objective et subjective». Il existe, écrivait-il, «deux modes distincts de pensée scientifique, l'un et l'autre fonction, non pas certes de stades inégaux de développement de l'esprit humain, mais de deux niveaux stratégiques où la nature se laisse attaquer par la connaissance scientifique: l'un approximativement ajusté à celui de la perception et de l'imagination, et l'autre décalé » (Lévi-Strauss, 1962, p. 28). Toutefois, mon enquête de terrain ne me conduit pas à pointer deux formes de pensée, mêlées dans la connaissance produite par les géologues, mais à déceler le rapport intime qui lie ces chercheurs à leur objet et qui dépasse la dualité du savoir ${ }^{9}$.

Comme les science studies $^{10}$, cet article traite de la «science en train de se faire ${ }^{11}$, pointe l'empreinte de ceux qui la font, et partage l'idée qu'il n'y a pas une science mais des sciences qui se distinguent par une diversité de pratiques. Mais il n'étudie pas les controverses qui animent la recherche et porte son attention sur le travail de terrain plutôt que sur celui de laboratoire ${ }^{12}$. Précisons enfin que les conclusions avancées reposent sur des observations lors de trois sorties de terrain dans les Alpes avec des chercheurs en sciences de la Terre en 2013, 2014 et 2015, et sur des entretiens menés principalement à Grenoble dans le laboratoire ISTerre, essentiellement auprès de géologues alpins ${ }^{13}$. Même si ces derniers ne se définissent pas tous comme des géologues de terrain, certains se destinant d'ailleurs entièrement à la modélisation, ils reconnaissent tous une place importante au terrain dans un travail qu'ils jugent collectif. Nous nous demanderons si ce n'est pas là un phénomène essentiel dans la production de connaissance en sciences de la Terre, mais opacifié par la place de plus en plus grande prise aujourd'hui par l'expérimentation et la modélisation dans les pratiques et l'argumentation scientifiques.

\footnotetext{
9 De même, «L'émotion n'exclut pas la raison» souligne l'historienne Arlette Farge: «Il n'est pas d'intelligence sans émotion, pas d'émotion sans intelligence [...] Se confronter à elle relève d'un véritable exercice intellectuel» (Farge, 2013, p. 100).

${ }^{10}$ Les science studies regroupent un ensemble de recherches en sciences humaines sur le travail, les pratiques et les méthodes scientifiques. Voir Pestre (2006).

${ }^{11}$ Latour distingue la science faite (la boîte noire) et la science en train de se faire, qu'il symbolise par les deux faces de Janus (Latour, 2005).

${ }^{12}$ L'article s'intéresse aussi à l'affect, laissé de côté par les sciences studies (Charvolin, 2009).

${ }^{13}$ D'autres échanges ou entretiens avec des participants non géologues au projet «géo-inspire» (chimistes ou ingénieurs) ont alimenté la recherche.
}

\section{Le rapport intime des géologues de terrain à leur objet}

\section{«... on a exhumé la chaîne de montagnes... »}

Dès la première sortie de terrain dans les Alpes en septembre 2013 dans le massif du Chenaillet (affleurement de croûte océanique) puis dans le massif de l'Oisans (veines métamorphiques), je fus frappée par la façon dont les chercheurs parlaient entre eux des phénomènes qu'ils observaient et de la forme narrative dont ils usaient pour me les expliquer. Cela s'est confirmé ensuite, lors des entretiens ou échanges spontanés avec les géologues, parfois au-delà même du laboratoire où se déroulait l'étude. Ce qu'ils étudient ne semble pas leur apparaître comme des phénomènes naturels extérieurs qu'ils chercheraient à décrire. Au contraire, un rapport qu' on peut qualifier d' « intime » à leur objet est perceptible à travers leur discours, qui les engage dans «l'histoire qu'ils racontent», pour reprendre des termes souvent entendus. Le temps qui les sépare des phénomènes qu'ils cherchent à élucider ne semble plus exister : ils parlent au présent lorsqu'ils expliquent des faits datant de millions ou de milliards d'années, comme en témoigne ce chercheur qui, me montrant la calcite sur le relief, dit : «Là, la roche est à 300 degrés $»$.

De plus, les phénomènes ne sont pas décrits sur un mode mécanique et distancé mais semblent associés à une volonté qui implique le chercheur. Un très grand nombre d'éléments de discours de différents géologues présents lors des sorties sur le terrain, dont quelques-uns sont retranscrits ci-dessous, en expriment la teneur:

«De l'eau a circulé quand on a fait l'alpin.»

«...vv la composition de la roche, c'est sûr qu'on a hydraté. »

«Quand on a exhumé la chaîne de montagnes...»

«On a extrait énormément de magma de la roche à pyroxène. »

« $\mathrm{Si}$ tu fais fondre ton manteau, tu n'auras pas de gabbro. »

«Le socle est très résistant. On a du mal à le plisser. »

"Quand on plisse, on a tendance à développer un plan de schistosité, de cisaillements des plans.»

«En comprimant le socle, on a créé des cassures et on a créé des veines.»

«... après on a rapproché les continents...»

Et lorsqu'un chercheur en questionne un autre pour avoir des précisions sur un phénomène qui s'est produit dans les temps géologiques, il l'implique: «Et tu cristallises la calcite sous l'effet de la pression dans ce cas-là ?». 
Une fois l'étonnement passé, j'émettais l'hypothèse que le travail en laboratoire, où les scientifiques font advenir des réactions naturelles afin d'éclairer ce qui a produit les phénomènes qu'ils observent sur le terrain, pouvait peut-être expliquer leur implication et l'usage du présent. Mais lorsque je les ai interrogés (dans des entretiens postérieurs ou lors d'échanges informels) sur ces formes du discours, je leur ai donné à voir quelque chose de si familier qu'ils ne l'avaient pas relevé. Ils expliquent alors ce «on » qui les engage (collectivement au moins) par la grande proximité qu'ils ont avec leur objet. Cette relation «presque intime» avec les roches les amènerait à personnaliser les phénomènes. Lors d'un entretien, un géologue de terrain me dit dans le même sens, que c'est l' « attachement » à son objet qui l'amène à vouloir se «l'approprier».

Cette personnalisation de l'objet étudié peut être perceptible dans la façon dont les minéraux sont nommés en sciences de la Terre. Si leurs noms font parfois référence à leur composition chimique (par exemple la sidérite, du grec sideros qui veut dire fer), ils sont dans de nombreux autres cas liés à l'histoire de leur découverte. L'oxyde ferreux, par exemple, ainsi nommé par les chimistes ( $\mathrm{FeO}$ avec $\mathrm{Fe}$ pour le fer et $\mathrm{O}$ pour oxygène) depuis la réforme de la nomenclature chimique en 1787, est de la «wüstite» pour les géologues qui utilisent la dénomination issue du métallurgiste allemand Fritz Wilhelm Friedrich Wüst. Mais ce n'est pas toujours le nom du découvreur du minerai qui inspire son appellation. Ce peut être aussi celui d'un scientifique à qui l'on veut rendre un hommage posthume. C'est le cas de la smithsonite, nom donné en 1832 à un minéral de carbonate de zinc en hommage à James Smithson, minéralogiste britannique qui en avait préalablement fait l'analyse chimique. Wihelm Karl von Haidinger, lui, a dédié la bornite au minéralogiste autrichien Ignaz von Born. Et l'on pourrait donner bien d'autres exemples.

C'est parfois l'apparence du minéral qui sera déterminante. L'olivine, découverte en 1790, tire son nom de sa couleur vert olive. Ce peut être la localité où le minéral fut découvert qui inspire son nom, comme l'Andalousite dont les échantillons étudiés par le naturaliste Jean-Claude Delamétherie en 1798 étaient supposés venir d'Andalousie (ils provenaient en réalité d'une autre région d'Espagne).

Dans les cas évoqués ici, comme dans de nombreux autres, c'est l'histoire "humaine» de ces minéraux qui est retracée, histoire indissociable de celle des connaissances que nous en livrent les sciences de la Terre. C'est assez frappant dans le cas du polonium, par exemple, qui tire son nom, non pas du lieu où il a été initialement prélevé par un scientifique, mais des origines polonaises de Marie Curie-Sklodowska, qui l'a découvert lors de ses premières recherches sur la radioactivité de la pechblende (minéral radioactif dont Pierre et Marie Curie ont plus tard extrait l'uranium). Dans le cas du graphite, c'est l'histoire sociale du minéral que transmet son nom. Il fut en effet nommé ainsi en 1789 par le minéralogiste allemand Abraham Gottlob Werner en référence au grec graphein signifiant écrire, parce qu'on l'utilisait depuis le XVII siècle pour fabriquer des mines de crayons à la place du plomb. On peut y voir le premier regard que porte le géologue sur le minéral, comme une entité qui n'est pas entièrement définie par sa composition chimique, car il est aussi attaché aux personnes qui ont contribué à en faire avancer la connaissance et au milieu dans lequel il a été observé et/ou prélevé sur le terrain.

\section{Au-delà de l'intuition première}

L'implication du chercheur, dont témoigne sa forme de langage, n'est pas seulement l'expression de son intuition $^{14}$, ce « flair scientifique » qui doit le conduire à «placer le regard dans la direction de ce qui se donne à comprendre » (Nouvel, 2000, p. 46) ${ }^{15}$. Elle n'est pas non plus son vécu subjectif qui stimule et guide sa recherche au-delà des premiers instants (la passion du scientifique $^{16}$, ce sentiment océanique que décrit Freud comme intense «récompense de tout réel progrès dans la compréhension $\gg{ }^{17}$ ) ou encore l'émotion de l'historien face à une archive ${ }^{18}$. L'implication, et même l'engagement du géologue dont il est question ici, ne renvoie pas à l'intuition initiale, ou à ses «émotions》 ou «passions» qui orientent le résultat final. Elle est, comme nous allons le voir, la condition d'existence de l'objet que le scientifique va étudier.

14 Pour Raymond L. Wilder, qui traite de l'intuition du mathématicien comme «educated guess», cette première étape est essentielle car, qu'elle se vérifie ou non, aucun progrès scientifique ne peut s'engager sans elle (Wilder, 1967).

${ }^{15}$ Une intuition souvent passée sous silence tant «seules les sciences qui prouvent, c'est-à-dire qui peuvent se prévaloir de faits faisant autorité sont dignes d'échapper à la disqualification» (Stengers, 2013, p.27). Celles-ci, sous le terme de «sound sciences » (sciences solidement étayées et validées par leurs pairs) aux États-Unis, rappelle Stengers (2013), ne peuvent donner de place à une intuition qui pourrait être assimilée à l'opinion.

${ }^{16}$ Reprenant Michael Polanyi, Nouvel insiste sur la fonction heuristique de la passion : un «sentiment d'extrême exaltation qui peut être ressenti par un scientifique au moment de la découverte » (Polanyi M., 1974, p. 134. Personal knowledge. Towards a post-critical philosophy, Chicago, the University of Chicago Press, repris dans Nouvel, 2000, p. 17).

17 Medawar P.B., 1979, p. 7. Advice to a young scientist, NewYork, Harper \& Row, p. 7, repris dans Daston et Galison, 2012, p. 361 .

18 Une archive qui ouvre entre lui et «l'objet visité une véritable interrogation, et donne envie d'expliquer une dimension inaccoutumée » (Farge, 2013, p. 100). 


\section{Un sujet engagé dans le savoir qu'il produit}

\section{L'échelle humaine}

Il ressort de l'enquête que l'objet de recherche du géologue n'est pas la roche en tant que telle mais cette roche prise dans son contexte particulier. Sur le terrain, le géologue va d'ailleurs commencer par observer de loin la géomorphologie du paysage afin d'identifier et de noter dans son carnet les couches ou strates, les filons, failles ou fractures, et d'en réaliser une cartographie. Ensuite seulement il ira sur l'affleurement pour l'étudier de plus près, en prélever un échantillon avec son marteau, puis en voir les détails avec sa loupe. C'est à l'échelle de son terrain que le géologue va pouvoir embrasser cette totalité pour en saisir toute la complexité, ce qu'un des chercheurs interviewés traduit par «l'échelle humaine» : un territoire qu'on peut arpenter à pied comme pour être sûr qu'on ne fragmente pas son observation.

«L'échelle à laquelle on peut travailler, c'est l'échelle humaine, ça fait $20-30 \mathrm{~km}$, on peut y aller en voiture. [...] On peut se promener d'une zone à l'autre, donc effectivement, sur une mission, on peut faire des grandes distances. Mais si vous faites des grandes distances, c'est-à-dire, par exemple, l'échelle de plusieurs centaines de kilomètres, vous ne ferez que des observations ponctuelles. Donc, après, on va essayer de faire le lien entre ces observations ponctuelles, il y a donc forcément de l'extrapolation. Maintenant, si on veut faire un travail sérieux, affiné, précis, l'échelle humaine, c'est effectivement quelque chose entre 30 et $50 \mathrm{~km}$ de long et $20 \mathrm{~km}$ de large. C'est cette zone-là, ça, on peut le faire à pied [...]. L'idéal, si on ne veut pas faire d'extrapolation, c'est d'observer complètement une zone et pas de faire des observations ponctuelles $(20-30 \mathrm{~km}) »$.

Ainsi, le chercheur est engagé physiquement sur le terrain comme il l'est symboliquement par son langage lorsqu'il emploie ce «on ». C'est la place du corps et des sens dans la production de connaissance qui est ici affirmée (à travers la marche), comme le précisait JeanClaude Kaufmann (1997) pour les connaissances plus ordinaires, où la décision et l'action paraissent impossibles sans cette «intelligence du corps». «La première erreur, écrivait-il, consiste à séparer sensations et cognition, corps et pensée; la seconde, à croire que les sensations sont périphériques ». Si ces «sensations» sont essentielles pour Kaufmann, c'est parce qu'elles peuvent tout autant permettre de fermer le champ des possibles ouvert par la pensée rationnelle ${ }^{19}$ que remettre en question ce qui était jusqu'alors admis en engageant cette pensée rationnelle dans une nouvelle direction. Ainsi, on ne sait pas «qui agit sur qui, les sensations sur

19 Par une opération de «réduction en phase finale du raisonnement» (Kaufmann, 1997, p. 305). les idées, ou les idées sur les sensations » (Kaufmann, 1997, p. 312).

Cet engagement du chercheur sur le terrain, de toute sa personne (associé à l' «attachement» que décrivait l'un d'eux) nous a conduit à qualifier d'intime sa relation à son objet. Le géologue, en parcourant à pied l'environnement qu'il cherche à expliquer, en s'impliquant dans les phénomènes passés qu'il décrit, devient partie prenante du milieu qu'il étudie et qu'il habite ${ }^{20}$. Si nous parlons d'ailleurs d'environnement, ce n'est précisément pas au sens de ce qui l'environne, mais de ce dans quoi il est pris : un environnement "vécu» ${ }^{21}$, que le terme de milieu traduit plus justement, comme nous le verrons plus bas.

Dès l'instant où il se fait observateur, il fait partie du monde qu'il étudie et, si l'on suit l'anthropologue Tim Ingold, c'est justement par cette relation dynamique à l'environnement qu'il peut le saisir et en produire une connaissance. La notion de "distance de sécurité» (Ingold, 2013, p. 29) que critique Ingold, et qui serait celle que chercherait à construire le scientifique pour assurer l'objectivité de son propos, ne se retrouve d'ailleurs pas chez le géologue de terrain. Bien au contraire, comme les citations que nous avons retranscrites en témoignent, la fiabilité de sa connaissance est pour ce dernier relative à la proximité de son objet d'observation. Ingold (2013, p. 38) insiste d'ailleurs de façon plus générale sur le fait que la compréhension des phénomènes par le scientifique « repose sur des aptitudes à la perception qui émergent, pour chaque être, à travers un processus de développement dans un environnement historique particulier ». Les perceptions, et l'environnement même qu'on cherche à connaître, ne se révèlent que dans «un mode d'engagement dans le monde», un «engagement actif» comme le décrit l'anthropologue (Ingold, 2013, p. 137).

Ainsi, il n'y a pas pour Ingold (2013, p. 145) de réalité extérieure (monde physique) qui serait source de données sensorielles brutes: «Les personnes et leurs environnements sont des composants mutuellement constitutifs du même monde ». Ce qui, dans notre cas, signifie que le géologue n'est pas indépendant de l'environnement auquel il nous donne accès. C'est sa capacité, que lui seul

\footnotetext{
20 Si le géophysicien habite aussi le terrain par ses capteurs, c'est dans un sens différent, de «marquage» du territoire. Même si ses capteurs peuvent être assimilés à des instruments exosomatiques, prolongeant ses capacités perceptives, la médiation technique qu'ils représentent produit une mise à distance du milieu (quel que soit son éloignement physique) que n'a pas le géologue de terrain.

21 Cette distinction entre les environnements «vécus» et l'Environnement, avec un E majuscule (celui du réchauffement climatique, par exemple) est développée dans Raineau, 2014.
} 
a, de par sa formation et son expérience propre, de voir les dynamiques passées dans le présent qui lui permet de donner existence à cet environnement particulier qu'il étudie, un paléo-environnement. Il devient «capable de percevoir des formes invisibles au profane» (Daston et Galison, 2012, p. 378).

\section{Le milieu}

C'est un «monde propre» auquel se donne accès le géologue, dans le sens où Jacob von Uexküll parle $\mathrm{d}^{\prime}$ umwelt $^{22}$. Bien sûr Uexküll tire sa théorie du monde animal: il montre que chaque espèce, bien que pouvant partager un même territoire, vit néanmoins dans un «milieu » particulier ou «monde propre» en fonction de ses capacités perceptives (de l'espace et du temps), liées à ses sens et aux actions qu'il mène dans son monde. Le milieu d'un animal forme ainsi une « unité close» qui lui est propre, c'est son monde perceptif et actantiel (Uexküll, 2010, p. 25). Deux sujets n'entretiendront pas la même relation avec les choses et avec le temps : le milieu d'un animal n'est pas l'environnement indifférencié dans lequel nous voyons des animaux (et des humains) qui partagent un même territoire ${ }^{23}$. C'est ici le terme d'environnement qui renvoie à quelque chose d'abstrait et d'inaccessible (comme l'est celui de nature pour Ingold): un ensemble de données qui seraient indépendantes du sens qu'il revêt pour les êtres qui l'habitent. Il n'y a pas de monde universel perceptible car percevoir suppose des êtres vivants qui ressentent, agissent et se projettent dans ce qui devient ainsi leur monde. Uexküll, pour étayer son approche phénoménologique, donne l'exemple du chêne qui constitue des multitudes de milieux différents selon ses habitants (renard, chouette, écureuil, fourmi, bostryche, ichneumon) ou selon le rôle variable qu'il joue pour les humains (Uexküll, 2010, p. 156 et suiv.). Si on voulait regrouper tous ces milieux, on ne reconstituerait pas le chêne en tant qu'objet mais on aurait un «chaos» écrit Uexküll. Aucun des sujets de ces milieux ne peut reconnaître le chêne comme entité supportant tous ces milieux (Uexküll, 2010, p. 162).

Il y a ainsi des milieux différents et non des perceptions différentes d'un monde universel qui formerait une unité de temps et de lieu. En extrapolant la théorie de Uexküll, on peut considérer que le géologue

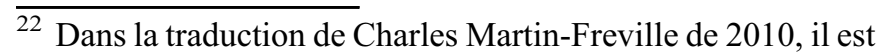
question de «milieu», là où on traduisait jusqu'alors «umwelt»par «monde propre» (Uexküll, 2010).

${ }^{23}$ Il existe des choses et des êtres dans les mondes perceptifs de certains, qui n'existent pas pour d'autres. Par exemple, le choucas ne voit pas la sauterelle immobile mais seulement en mouvement. Uexküll (2010, p. 88-89) dit que la sauterelle au repos n'existe pas dans le monde perceptif du choucas.
}

développe par ses études et sa pratique un système perceptif singulier, qui passe en grande partie par la vue, même si l'odorat fut aussi évoqué lors d'entretiens ${ }^{24}$. Daston et Galison (2012, p. 378) insistent sur cet «œil entraîné », déterminant dans la formation du géologue dès le $\mathrm{XX}^{\mathrm{e}}$ siècle, et citent à titre d'exemple l'atlas minéralogique d'Oskar Oelsner en 1961 «qui exerçait le géologue en herbe à classer des échantillons de minerai microscopique ». Ils pointent que ce tournant s'est opéré avec de «nouvelles méthodes de formation des étudiants du supérieur en science, pour voir, manipuler et mesurer, une calibration de l'esprit, de la main et de l'œil peut-être sans précédent dans sa rigueur et sa portée » (Daston et Galison, 2012, p.376). Le géologue devient alors sensible à certains éléments et insensible à d'autres, et crée par ce biais son monde perceptif propre. Il perçoit le sous-sol à travers l'observation des affleurements et visualise la dynamique qui conduit à la formation des roches présentes. Il reconnaît à l'œil nu, ou par quelques gestes simples, un grand nombre d'espèces minérales: leurs forme, couleur, texture, structure ou disposition relative sont pour lui des indicateurs essentiels. Son système perceptif est prolongé par des instruments opérant pour lui comme exo-somatisation (boussole avec clinomètre permettant les mesures topographiques, marteau pour casser une roche et mieux voir les minéraux qui la composent, loupe, carte, etc.). Là où moi j'observais des géologues observant des paysages, des montagnes, des roches, ces géologues voyaient plissements, pendage, litage et débit de la roche. De même, le géologue perçoit la végétation comme un élément relatif à la composition des roches là où l'écologue verra tout autre chose.

Ainsi, en s'engageant différemment sur un même terrain, en raison de leur formation, de leur expérience et de leurs outils, un géologue, un écologue, un biologiste, un chimiste, un sociologue ou un anthropologue produiront différents «mondes propres» et par là proposeront chacun une connaissance spécifique. Mais ce sont chaque fois des expressions de la totalité d'une réalité perçue et non des éléments partiels qui en s'additionnant produiraient, tel un puzzle, la connaissance d'un monde universel. Uexküll ouvre d'ailleurs en conclusion son analyse dans ce sens et soutient qu'aucun naturaliste (il fait référence aux biologistes, zoologistes, botanistes, astronomes ou physiciens) ne saisit la «nature» en tant qu' «Un». Ce «Un» supporte pourtant tous les milieux que nous décrivent les naturalistes, dit Uexküll, mais il reste à jamais inatteignable. Et aucune connaissance ne peut provenir de l'agencement des

\footnotetext{
${ }^{24}$ La présence d'une odeur inhabituelle a été évoquée lors de la description par un géologue d'une expérience en laboratoire, celle-ci ayant conduit à réorienter l'expérience. Mais, a-t-il précisé, «Ça, on ne l'écrit pas dans un article».
} 
propriétés de ces milieux. "Derrière tous ces mondes qu'il [le scientifique] produit se cache, manifestement à jamais, leur sujet: la nature », écrit-il pour clore son ouvrage (p. 166). Uexküll fait ainsi lui-même le pont entre son umwelt, le milieu animal, et le fûdo de Tetsurô Watsuji, le milieu humain ${ }^{25}$. Augustin Berque (2014) souligne d'ailleurs combien ces deux auteurs posent, l'un en éthologie, l'autre en philosophie, quasiment au même moment les principes au cœur de la mésologie telle qu'on l'entend aujourd'hui, c'est-à-dire comme la science des milieux (l'écologie étant la science de l'environnement).

Ce qui importe ici est de comprendre que ce qu'étudie le chercheur n'est pas un donné extérieur puisque c'est seulement en y étant engagé, en formant un tout indivisible avec lui, qu'il le fait advenir et peut en avoir connaissance. Le milieu n'est toutefois pas une fusion entre sujet et objet qui les ferait disparaître tous les deux. Il y a bien «un rapport d'altérité dans la production de savoir », comme le définit Sophie Poirot-Delpech (2013, p. 105) pour les sciences sociales, par le « lien de réciprocité qui se tisse sur le terrain ». C'est ce qui conduit certains à militer en faveur du maintien de l'idée de nature, même s'il faut la comprendre comme un ensemble hétérogène dont les humains ne sont pas séparables ${ }^{26}$. Un naturel ou un «sauvage» qui sans être extériorité est une altérité qui résistera à celui qui chercherait à le contrôler, le maitriser ou le piloter. Quelque chose échappe en effet toujours au chercheur car, qu'il soit en sciences de la nature ou en sciences sociales, son objet (comme la relation qui le fait advenir) est en perpétuel devenir.

\section{Le rôle du terrain dans les sciences de la Terre}

\section{Saisir la complexité}

Même si l'observation sur le terrain ne peut pas se suffire à elle-même, et est parfois qualifiée de « poétique » par des géologues plus enclins à faire des modèles ${ }^{27}$, elle reste toutefois une étape à laquelle ne peuvent se substituer ni l'expérimentation ni la modélisation. Elle permet de ne pas dissocier un phénomène singulier de son contexte et de

\footnotetext{
${ }^{25}$ C'est A. Berque qui, dans sa traduction de Tetsurô Watsuji, traduit fûdo par «milieu» et rapproche Watsuji de Uexküll, dans un lien à Heidegger (Watsuji, 2011).

${ }^{26}$ «Le concept de nature sauvage est efficace, il borne, il oppose et permet de penser» (Devictor, 2015, p. 91).

27 Les géologues de terrain sont parfois, avec humour et bienveillance, qualifiés de «poètes» par leurs collègues expérimentateurs ou modélisateurs, ce qui, à leurs yeux peut-être, les éloignerait de la rigueur de la démonstration ou les ramènerait à une pratique contemporaine du romantisme.
}

ne pas oublier le maillage et l'histoire dont il est issu et dont il fait partie. Elle rappelle la complexité de l'objet du géologue, complexité qu'Edgar Morin (1990, p. 21) définit comme «ce qui est tissé ensemble», un tissu «de constituants hétérogènes inséparablement associés ». La réalité nous apparaît pour cela comme un ensemble inextricable, ambigu, incertain et la pensée complexe « sait au départ que la connaissance complète est impossible», l'omniscience inatteignable (Morin, 1990, p. 11). Une complexité que le chercheur en laboratoire a conscience de ne pas saisir par son expérimentation, ou que le modélisateur n'est jamais certain de correctement refléter. Le contexte dans lequel l'échantillon fut extrait ne doit pas être oublié par l'expérimentateur et relativise toute expérience postérieure, comme l'évoque un géologue lors d'un entretien : «Parce qu'on a un "caillou", prélevé dans la nature, on peut penser que c'est la réalité et on perd en lucidité»".

Ce que cherche à éviter ce chercheur, c'est ce que Morin (1990, p. 11) appelle «l'intelligence aveugle ${ }^{28} »$, qui «isole ce qu'elle sépare, et occulte tout ce qui relie, interagit, interfère ». Elle conduit à « déchirer et morceler le tissu complexe des réalités, et donner à croire que le découpage arbitraire opéré sur le réel était le réel luimême» (Morin, 1990, p. 19). Ayant cette culture du terrain (de par sa formation), c'est sans doute conscient de cette limite que le géologue appréhende dans le laboratoire l'expérimentation qui permet de décomposer des processus et des éléments pour voir comment chacun interagit avec l'autre. Elle ne peut pas reproduire la complexité du terrain mais permet d'obtenir des résultats précis sur certains processus. Le modélisateur remettra ensuite ensemble ce qui a été dissocié, pour (re)créer une complexité et intégrer des échelles de temps et d'espace qui ne sont pas celles qu'on peut embrasser sur le terrain et auxquelles ne peut pas prétendre l'expérimentation.

Le modèle est d'ailleurs aussi soumis à la réalité du terrain. Ainsi, «c'est le terrain qui a le dernier mot, toujours » insistait un chercheur, en ajoutant plus tard: «La preuve de terrain est quasiment irréfutable, c'est l'évidence». Un autre l'exprimait en précisant : «Il y a des gens qui font de la modélisation et qui, à un moment donné, arrivent à modéliser plein de choses, c'est-à-dire qu'ils rentrent des paramètres, ils sortent des résultats, et ils viennent te dire "Est-ce que, dans la nature, ça existe, ce que j'ai modélisé ?" »

La «nature», même si elle n'existe que comme milieu pour l'observateur, paraît donc partie prenante de la «science en train de se faire » tout au long du travail du

28 Faisant écho à la «vision aveugle» de l'objectivité mécanique qui caractérise l'éthique et la pratique scientifiques au milieu du XIX ${ }^{\mathrm{e}}$ siècle selon Daston et Galison (2012). 
géologue, contrairement à ce qu'analyse Latour (2005) dans la recherche de laboratoire, où il décrit qu'elle ne joue qu'un rôle de justification ex post des résultats (dans la « science faite »), une fois la controverse éteinte ${ }^{29}$. Si on se réfère à d'autres travaux de l'auteur, on constate effectivement que le terrain n'a pas le même sens et la même portée dans toutes les disciplines. Ainsi, lorsque Latour (2007) relate le travail du botaniste et du pédologue dans la forêt de Boa Vista, il nous donne à voir un rapport assez différent au terrain. C'est, en effet, en sortant les plantes de leur contexte local (la forêt) et en les agençant selon des principes qui lui sont propres, en les classant, les rangeant, les nommant, que le botaniste peut les analyser : «En perdant la forêt, on gagne le savoir sur elle » ponctue Latour, notant que le travail du chercheur ne diffère pas dans ce cas de celui qu'il peut mener dans le laboratoire (Latour, 2007, p. 184). De même, lorsque le pédologue isole une parcelle de sol de son contexte « trop complexe» en la délimitant physiquement avec le topofil, il la transforme en «protolaboratoire» (Latour, 2007, p. 190). Il semble que dans ces deux exemples, l'inventaire et la collecte de spécimens deviennent les objectifs essentiels du travail de terrain. Toutefois, si en sortant une partie du sol de son continuum, et en collectant et classant des mottes de terre dans son pédocomparateur, le pédologue la change d'état, puis de lieu, il produit aussi chaque fois un nouveau milieu, au sens d'une nouvelle relation entre lui et l'objet ainsi transformé. Cette notion de milieu s'étend donc audelà du terrain et peut exprimer ce qui lie un chercheur à son objet, fût-ce dans le laboratoire ${ }^{30}$, à la différence que, dans ce cas, des éléments sont abstraits du contexte, contexte que le géologue de terrain cherche précisément à saisir.

\section{La complémentarité des métiers en sciences de la Terre}

Le travail de terrain et celui de laboratoire et de modélisation coexistent, mais ils ne s'opposent pas dans la culture du géologue, ils se complètent, se répondent et ne paraissent pas former des approches concurrentes. L'observation des affleurements fait partie de la culture du géologue même si la spécialisation des tâches aujourd'hui voue certains à la modélisation presque exclusivement. Cela permet à tous, expérimentateurs ou modélisateurs ${ }^{31}$, de reconnaitre la place de cette connaissance de terrain

\footnotetext{
29 «Tant qu'a duré la controverse, aucun appel à la nature ne pouvait apporter de l'eau au moulin de l'un des deux camps... Du moment où le débat s'est arrêté, le supplément de force fourni par la nature explique rétrospectivement le fait qu'il y ait été mis fin» (Latour, 2005, p. 238).

${ }^{30}$ Nous renvoyons ici à l'article de Charlotte Brives décrivant les interactions entre humains et levures dans le laboratoire (Brives, 2017).

${ }^{31} \mathrm{~S}$ 'ils ont une formation en géologie et ne viennent pas de la physique.
}

qui passe par les sens et l'engagement du chercheur) dans le savoir scientifique qu'ils produisent. Ainsi, un géologue « naturaliste » exprime la complémentarité entre l'observation et la modélisation:

«Aller sur le terrain, je pense que c'est la base, parce que la géologie, quand même, l'objet géologique, c'est l'affleurement. Donc ça, pour moi, c'est ce qu'il y a de plus important. Il faut être capable d'aller sur le terrain, de reconnaître ces roches, de comprendre les associations entre les différents types de roche, de comprendre un contexte et puis ensuite, de raconter une histoire à partir de ça. Mais pour raconter cette histoire, il faut connaître le type de roche, donc il faut peut-être faire des analyses, il faut peutêtre avoir des contraintes chronologiques, donc des datations, il faut ensuite peut-être changer d'échelle, pour essayer de voir comment se localise la déformation. Et puis ensuite, au fur et à mesure, il y a des nouvelles questions qui se posent et puis ensuite, on propose un modèle. Et ce modèle est enrichi de données qui sont peut-être des données expérimentales, des données géophysiques, etc. Et on va vers un modèle qui permet d'expliquer au mieux ce qu'on observe dans la nature. Le but, c'est un peu ça. Mais les deux ne sont pas opposés.»

Si le terrain est le point de départ, ce n'est donc pas dans une vision linéaire de la recherche où il fournirait des données au modélisateur et des matériaux à l'expérimentateur. Un géologue exprime dans un entretien le va-etvient de la recherche : «C'est là où l'on peut faire le retour sur les données en disant: "Oui, quand même, il faut que l'on creuse là parce que là, le modèle dit que c'est une piste à creuser". Et donc là éventuellement, on peut refaire la boucle complète».

Le terrain permet toujours d'appréhender un phénomène sans le détacher du milieu dans lequel on l'observe. En ce sens, un géologue naturaliste nous dit saisir le « général », là où le géologue de laboratoire s'intéressera au « particulier ». «L'expertise de terrain, c'est d'aller, je ne sais pas, par exemple...une chaîne de montagnes, c'est de pouvoir en fait décrire les affleurements, comprendre l'association des différents types de roche, et puis remonter à un contexte. Donc on va du général au particulier. Et quand on arrive au particulier, on a besoin de faire de l'expérimental».

Le chercheur a d'abord accès à ce général visuellement en regardant l'affleurement à distance, à une échelle où on peut appréhender la trame dans laquelle sont tissés les éléments observés. Et si le géologue dit «raconter une histoire» à partir de son observation, c'est sans doute justement parce qu'il peut mettre en mouvement ces éléments qu'il perçoit dans leur complexité, pour donner à voir la dynamique des phénomènes. Travailler à l'échelle humaine, l'échelle des sens, n'est pas se concentrer sur le particulier, mais chercher au contraire à embrasser ce qui fait de ce «milieu» une totalité. C'est cette « reconnaissance holistique» que pointent Daston et Galison (2012, 
p.382 et p.387) et qui permet de saisir «l'apparence générale ».

\section{Conclusion}

Comme nous le précisions en introduction, le projet dans le cadre duquel notre étude s'est déroulée adressait aux sciences humaines une interrogation portant sur la posture du géologue face aux problèmes environnementaux d'aujourd'hui. Elle supposait que les représentations et les pratiques du chercheur étaient à interroger comme celles de tous les autres acteurs sociaux. Et face à cela se posait sous un nouveau jour la question de l'enjeu et de la forme que pouvait prendre la collaboration entre disciplines dans un cadre de transition énergétique et écologique. L'analyse du rapport du géologue au terrain que nous avons développée ici permet d'avancer ou d'amorcer la réflexion sur ces deux points.

Qualifier de «milieu » ce qu'observe le chercheur sur le terrain permet d'abord de comprendre qu'il n'y a pas «d'objet neutre » et extérieur qui pourrait devenir objet commun de différentes disciplines. La spécificité de l'expérience et de la culture scientifique qu'un chercheur acquiert dans son domaine lui donne accès à un milieu particulier, qui ne peut pas être considéré comme un élément d'un tout qu'il s'agirait de reconstituer par l'addition des savoirs produits. Le risque aujourd'hui, notamment face à la problématique de l'Anthropocène, est d'oublier l'incommensurabilité et l'irréductibilité des milieux, même enchevêtrés, que reflètent ces savoirs ${ }^{32}$. Ce qui est donc en jeu, et que nous permet d'appuyer l'approche par la notion de milieu, est la pluralité du monde que la diversité des connaissances, des méthodologies et des objets (disciplinaires) doit permettre de conserver, tout comme on cherche à maintenir une diversité du vivant et des cultures. Comme le soutiennent Daston et Galison, «une pluralité de visions de la connaissance, comprises dans une acception très large comme différentes formes de fidélité à la nature, est probablement amenée à demeurer une caractéristique permanente de la science» (Daston et Galison, 2012, p.426). Elle devrait aussi conduire à une certaine prudence quant à l'usage qui peut être fait des connaissances scientifiques puisque nous ne pouvons accéder à la nature comme «Un», comme le soulignait Uexküll.

Dans leur étude des atlas scientifiques dans l'histoire des sciences naturelles, Daston et Galison montrent que depuis le début du $\mathrm{XX}^{\mathrm{e}}$ siècle, le «jugement exercé » du

\footnotetext{
${ }^{32}$ Bernadette Bensaude-Vincent et Sacha Loeve (2018, p. 376) insiste sur la multiplicité des temporalités qu'on ne saurait emboîter. L'enjeu est aujourd'hui d'« échapper au piège du temps unique qu'entretient le concept d'Anthropocène».
}

chercheur est à l'origine des images scientifiques produites. Loin de s'effacer, le scientifique engage donc son savoir et son expérience pour produire l'image qu'il juge la plus fidèle possible, de même que le géologue de terrain s'implique dans le milieu qu'il étudie pour pouvoir nous le décrire. «L'éthique de l'oubli de soi», qui était de rigueur pour assurer «l'objectivité mécanique » de la connaissance scientifique depuis le milieu du XIX ${ }^{\mathrm{e}}$ siècle, ne tient plus (Daston et Galison, 2012, p. 149).

Dès l'instant où le savoir produit n'est plus considéré comme indépendant du sujet connaissant, il devient important, et même nécessaire, de s'interroger sur l'ethos de la communauté scientifique à laquelle il appartient, c'est-à-dire à l'ensemble de ses valeurs, croyances et pratiques ${ }^{33}$. L'enjeu de la collaboration entre sciences de la nature et sciences sociales n'est-il pas dès lors de permettre cette réflexivité et de réfléchir, avant tout projet supposé les rassembler, au rapport que chacune entretient à son objet? Car, comme le définissent Prigogine et Stengers (1980, p. 185) ${ }^{34}$, la posture du chercheur, les questions qu'il se pose et les voies qu'empruntera sa recherche ne sont pas indépendantes des conceptions du monde à un moment donné dans la société ni des «angoisses» ou «hantises» qui s'y expriment. Et cette conception du monde sera en retour entretenue dans la société par les résultats qui seront issus de sa recherche. Les faits scientifiques qui seront énoncés dans ce contexte ont en ce sens une portée politique.

C'est bien cette réflexivité, sur la posture du géologue, que visait le projet géo-Inspire dans le cadre duquel s'est faite la recherche exposée ici. Par ce projet, s'inspirant de processus naturels pour permettre de recycler et de retraiter des déchets anthropiques, s'engageait aussi une réflexion sur le rapport des sciences de la Terre à leur objet. En proposant de faire du milieu naturel une source d'inspiration, il s'agissait de ne plus réduire la nature à un ensemble de ressources. $\mathrm{La}$ contribution à la transition énergétique ne devait donc pas s'opérer sur la seule perspective d'optimisation d'un processus de production d'hydrogène à partir de déchets industriels, mais portait aussi sur les représentations, normes morales et pratiques du chercheur, celles-ci participant à la nécessaire transformation du rapport de notre société à la nature.

\footnotetext{
33 Voir Robert King Merton, 1973.

34 Prigogine et Stengers (1980, p. 46) affirmant «l'interaction forte entre les questions produites par la culture et l'évolution conceptuelle de la science au sein de cette culture $»$.
} 


\section{Références}

Bensaude-Vincent B., 2016. Comment sortir du piège de la flèche du temps, Revue française d'éthique appliquée, 2, 2, 90-98, http://dx.doi.org/10.3917/rfeap.002.0090.

Bensaude-Vincent B., Loeve S., 2018. Penser Carbone, in Beau R., Larrère C. (Eds), Penser l'Anthropocène, Paris, Presses de Sciences Po, 375-389.

Berque A., 2014. La mésologie, pourquoi et pour quoi faire?, Nanterre, Presses universitaires de Paris Ouest.

Bonneuil C., Fressoz J.-B., 2013. L'événement anthropocène. La Terre, l'histoire et nous, Paris, Seuil.

Brives C., 2017. Que font les scientifiques lorsqu'ils ne sont pas naturalistes ?, Le cas des levuristes, L'Homme, 222, 3556, http://dx.doi.org/10.4000/lhomme.30143.

Charvolin F., 2009. Comment penser les sciences naturalistes «à amateurs» à partir des passions cognitives, Natures Sciences Sociétés, 17, 2, 145-154, http://dx.doi.org/ 10.1051/nss/2009027.

Daston L., Galison P.L, 2012. Objectivité, Dijon, Les Presses du réel.

Devictor V., 2015. Nature en crise. Penser la biodiversité, Paris, Seuil.

Dubey G., 2013. Introduction au numéro thématique «Embarqué», Socio-anthropologie, 27, 9-20, http://dx. doi.org/10.4000/socio-anthropologie.1403.

Farge A., 2013. La part de l'émotion, Socio-anthropologie, 27, 99-101, http://dx.doi.org/10.4000/socio-anthropolo gie. 1498 .

Ingold T., 2013. Marcher avec les dragons, Bruxelles, Zones sensibles.

Kaufmann J.-C., 1997. Le cour à l'ouvrage. Théorie de l'action ménagère, Paris, Nathan.

Latour B., 1991. Nous n'avons jamais été modernes: essai d'anthropologie symétrique, Paris, La Découverte.
Latour B., 2005 [1 ${ }^{\text {re }}$ éd. 1989]. La science en action. Introduction à la sociologie des sciences, Paris, La Découverte.

Latour B., 2007 [1 ${ }^{\text {re }}$ ed. 1993]. Le «pédofil» de Boa Vista- montage photo-philosophique, in Latour B., Petites leçons de sociologie des sciences, Paris, La Découverte, 171-225.

Lévi-Strauss C., 1962. La pensée sauvage, Paris, Plon.

Merton R.K., 1973. The sociology of science. Theoretical and empirical investigations, Chicago, University of Chicago Press.

Morin E., 1990. Introduction à la pensée complexe, Paris, ESF.

Nouvel P., 2000. L'art d'aimer la science, Paris, Presses universitaires de France.

Oreskes N., 2015. How earth science has become a social science, Historical Social Research, 40, 2, 246-270, http:// dx.doi.org/10.12759/hsr.40.2015.2.246-270.

Pestre D., 2006. Introduction aux Science Studies, Paris, La Découverte.

Poirot-Delpech S., 2013. La traversée des apparences, Socioanthropologie, 27, 103-111, http://dx.doi.org/10.4000/ socio-anthropologie.1499.

Prigogine I., Stengers I., 1980. La Nouvelle Alliance. Métamorphose de la science, Paris, Gallimard.

Raineau L., 2014. Innover autrement. La technique face à la crise environnementale, in Rochon F. (Ed.), Habitat et transition énergétique, Paris, l'Harmattan, 49-69.

Stengers I., 2013. Une autre science est possible! Manifeste pour un ralentissement des sciences, Paris, La Découverte.

Uexküll J. von, 2010 [1 $1^{\mathrm{re}}$ éd. 1956]. Milieu animal et milieu humain, Paris, Payot et Rivages.

Watsuji T., 2011 [1 ${ }^{\text {re }}$ éd. 1935]. Fûdo. Le milieu humain, Paris, CNRS éditions.

Wilder R.L., 1967. The role of intuition, Science, 156, 3775, 605-610, http://dx.doi.org/10.1126/science.156.3775.605.

Citation de l'article : Raineau L. Regard socio-anthropologique sur la géologie de terrain en sciences de la Terre. Nat. Sci. Soc. $28,3-4,260-269$. 